\title{
CONVERGENCE OF LINEAR AND NONLINEAR PADÉ APPROXIMANTS FROM SERIES OF ORTHOGONAL POLYNOMIALS
}

BY

D. S. LUBINSKY ${ }^{1}$ AND A. SIDI

\begin{abstract}
Analogues of the Nuttall-Pommerenke theorem and Wallin-type theorems for classical Padé approximants, are proved for linear and nonlinear Padé approximants formed from series of orthogonal polynomials, corresponding to a distribution $d \alpha(x)$ with at most finitely many sign changes.
\end{abstract}

1. Introduction. Let $\alpha(x)$ be a real function, defined in the interval $I=(a, b)$, (finite or infinite) such that the distribution $d \alpha(x)$ has at most finitely many sign changes in $I$-say $\mu$ many - and such that $\alpha(x)$ assumes infinitely many values in $I$. We assume there exists a sequence of real polynomials $\phi_{j}(x), j=0,1,2, \ldots$, such that $\phi_{j}$ is of degree exactly $j$ and is normalized so that

$$
\int_{I} \phi_{i}(x) \phi_{j}(x) d \alpha(x)= \begin{cases}0 & \text { if } i \neq j, \\ \pm 1 & \text { if } i=j\end{cases}
$$

A consequence of (1.1) is that for some constants $A_{i j k}$, we have

$$
\phi_{i}(z) \phi_{j}(z)=\sum_{k=|i-j|}^{i+j} A_{i j k} \phi_{k}(z) .
$$

We shall refer to the following lemma in the sequel (compare Cheney [ 2 , p. 110], Stahl [21, pp. 134-135]).

LEMMA 1.1. Let $f(x)$ be a function integrable in I. If $k \geqslant \mu$ and

$$
\int_{I} f(x) \phi_{i}(x) d \alpha(x)=0, \quad i=0,1,2, \ldots, k,
$$

then $f(x)$ either has at least $k-\mu+1$ sign changes in I or has infinitely many zeroes in $I$.

Proof. Let $\xi_{1}<\xi_{2}<\xi_{3}<\cdots<\xi_{\mu}$ be those points in $I$ at which $d \alpha(x)$ changes sign, and define $\psi(x)=\prod_{i=1}^{\mu}\left(x-\xi_{i}\right)$. Then $d \beta(x)=\psi(x) d \alpha(x)$ is a distribution having only one sign in $I$, and $\beta(x)$ has infinitely many points of change. Suppose that for some integer $\nu$ satisfying $0 \leqslant \nu<k-\mu+1, f(x)$ changes its sign precisely at the $\nu$ points $\eta_{1}<\eta_{2}<\cdots<\eta_{\nu}$ in $I$. Define $P(x)=\prod_{i=1}^{\nu}\left(x-\eta_{i}\right)$. Then $P(x) f(x)$

Received by the editors June 2, 1982.

1980 Mathematics Subject Classification. Primary 30E05, 30E10, 41A21.

${ }^{1}$ Research of this author supported by Lady Davis Fellowship. 
is of one sign in $I$. Further, as the polynomial $P(x) \psi(x)$ has degree $\nu+\mu \leqslant k,(1.3)$ gives

$$
0=\int_{I} f(x) P(x) \psi(x) d \alpha(x)=\int_{I} f(x) P(x) d \beta(x) .
$$

As $f(x) P(x)$ and $d \beta(x)$ have one sign in $I$, it follows that $f(x) P(x)$ must vanish almost everywhere with respect to $d \beta(x)$ and so $f(x)$ has infinitely many zeroes in $I$.

COROllaRY. $\phi_{k}$ has at least $k-\mu$ distinct zeroes in $I$, for $k \geqslant \mu$.

For more information on $\phi_{k}$, in the case when $\mu \geqslant 1$, see Stahl [21, pp. 134-136]. Of course the case $\mu=0$ is included in our results.

DEFINITION 1.1. Let $f(x)$ be such that the integrals $f_{j}=\int_{I} f(x) \phi_{j}(x) d \alpha(x)$, $j=0,1, \ldots$, all exist. Given nonnegative integers $L, M$, the linear Padé approximant $[L / M](z)$ to $f(x)$ is defined to be a rational function $P_{L}(z) / Q_{M}(z)$, where $Q_{M}(z)$ $\equiv 0$ and $P_{L}(z)$ are polynomials of degrees at most $M$ and $L$ respectively, such that

$$
\int_{I}\left(Q_{M}(x) f(x)-P_{L}(x)\right) \phi_{i}(x) d \alpha(x)=0, \quad i=0,1, \ldots, L+M,
$$

that is $Q_{M} f-P_{L}$ is orthogonal to all polynomials of degree at most $L+M$. The nonlinear Padé approximant $\langle L / M\rangle(z)$ to $f(x)$ is defined to be a rational function $P_{L}^{*}(z) / Q_{M}^{*}(z)$ where $Q_{M}^{*}(z) \neq 0$ in $I$ and $P_{L}^{*}(z)$ are polynomials of degrees at most $M$ and $L$ respectively, such that

$$
\int_{I}(f(x)-\langle L / M\rangle(x)) \phi_{i}(x) d \alpha(x)=0, \quad i=0,1, \ldots, L+M,
$$

that is, $f-\langle L / M\rangle$ is orthogonal to all polynomials of degree at most $L+M$.

Remarks. (a) Using (1.2), it is not difficult to see that (1.4) gives rise to $L+M+1$ linear equations in the $L+M+2$ parameters of $[L / M](z)$, and that only $f_{j}, 0 \leqslant j \leqslant L+2 M$, enter these equations. Similarly it can be seen that (1.5) gives rise to $L+M+1$ nonlinear equations in the $L+M+2$ parameters of $\langle L / M\rangle(z)$ and that only $f_{j}, 0 \leqslant j \leqslant L+M$, enter these equations. Furthermore, as stated in Cheney [2,pp. 178-179], the linear Padé approximants always exist. But it is not true that the nonlinear Padé approximants always exist. As for uniqueness, $[L / M]$ or $\langle L / M\rangle$ are not known to be unique in general. When $\alpha^{\prime}(x)>0$ almost everywhere in $I$ and $f(x)$ is real in $I$, uniqueness of $\langle L / M\rangle(z)$ was proved by Sidi [20].

(b) We note that the linear Padé approximants were introduced by Maehly [16] for the case $\phi_{j}=T_{j}$ where $\left\{T_{j}\right\}$ are the Chebyshev polynomials. Subsequently Cheney [2] defined linear Padé approximants from series of general orthogonal polynomials and Holdeman [11] considered more general approximations. Fleischer [5] applied the linear Padé approximants from Legendre polynomial series to some scattering problems in nuclear physics. Later Fleischer [6] introduced the nonlinear Padé approximants from Legendre polynomial series and gave a method for constructing them, which makes use of the fact that orthogonal polynomials satisfy the relations 
in (1.2). Clenshaw and Lord [4] introduced the nonlinear Padé approximants from Chebyshev series (Chebyshev-Padé table) and gave a recursive method for computing them. Sidi [19] gave another recursive method for computing these approximants, which complements the method of Clenshaw and Lord [4]. Later Gragg [9] considered some convergence properties of the Chebyshev-Padé table. Recently Chisholm and Common [3] have considered Padé-type approximations from Chebyshev series. Suetin [22] was the first to prove a general convergence theorem-namely an analogue of the de Montessus de Balloré theorem, as well as convergence in capacity of general nondiagonal Padé approximations under the assumption $\alpha^{\prime}(x)>0$ almost everywhere in $I$. However the authors have been informed by the referee that Suetin's proofs are incomplete as they are based on incorrect results of Rahmanov. Here the authors prove analogues of the NuttallPommerenke theorem and Wallin-type theorems under weaker assumptions on $\alpha$. They also prove a de Montessus de Balloré type theorem under weaker assumptions on $\alpha$, but with more restrictions on the functions involved.

Definition 1.2. For each $n \geqslant 1$, let $\mathbf{P}_{n}$ denote the class of polynomials of degree $n$, leading coefficient 1 . For a compact set $E \subset \mathbf{C}$, we define its logarithmic capacity $\operatorname{cap}(E)$ by

$$
\operatorname{cap}(E)=\lim _{n \rightarrow \infty}\left(\min _{P \in \mathbf{P}_{n}} \max _{z \in E}|P(z)|\right)^{1 / n},
$$

and extend the definition to noncompact $F$ by

$$
\operatorname{cap}(F)=\sup \{\operatorname{cap}(E) \mid E \subset F, E \text { compact }\} .
$$

See for example Hille [10, Chapter 16] for logarithmic capacity.

Definition 1.3. Given $m \geqslant 1, \varepsilon>0$, we use $\mathcal{L}(m, \varepsilon)$ to denote a lemniscate of the form $\left\{z|| P(z) \mid<\varepsilon^{n}\right\}$ where $1 \leqslant n \leqslant m$ and $P \in \mathbf{P}_{n}$. If $m=0$ or $\varepsilon=0$, $\varrho(m, \varepsilon)$ denotes the empty set. Given lemniscates $\mathcal{L}\left(m_{k}, \varepsilon_{k}\right), k=0,1,2, \ldots$, we set

$$
\underset{k}{\lim \sup } \mathscr{L}\left(m_{k}, \varepsilon_{k}\right)=\bigcap_{n=1}^{\infty} \bigcup_{k=n}^{\infty} \mathcal{L}\left(m_{k}, \varepsilon_{k}\right),
$$

that is, $\lim _{k} \sup \mathcal{L}\left(m_{k}, \varepsilon_{k}\right)$ is the set of points which belong to infinitely many of the e $\left(m_{k}, \varepsilon_{k}\right)$.

Note that $\operatorname{cap}(\mathscr{L}(m, \varepsilon))=\varepsilon$ (Hille [10]). See [14] for a study of sets of the form (1.6).

Definition 1.4. For any complex function $f(z)$, we define $\hat{f}(z)=\overline{f(\bar{z})}$, where, if $f(z)$ is analytic at $z_{0}$ with Taylor series $\sum_{n=0}^{\infty} a_{n}\left(z-z_{0}\right)^{n}$, then $\hat{f}(z)$ is analytic at $\bar{z}_{0}$ with Taylor series $\sum_{n=0}^{\infty} \bar{a}_{n}\left(z-\bar{z}_{0}\right)^{n}$.

Of course, if $f(z)$ is real in $\mathbf{R}$, then $\hat{f}(z) \equiv f(z)$.

Definition 1.5. Given the formal orthogonal polynomial series $U(z)=$ $\sum_{j=0}^{\infty} a_{j} \phi_{j}(z)$ and integers $0 \leqslant m \leqslant n \leqslant \infty$, we set

$$
[U]_{m}^{n}=\sum_{j=m}^{n} a_{j} \phi_{j}
$$


When $m$ (respectively $n$ ) is omitted, the lower (respectively upper) limit is taken to be 0 (respectively $\infty$ ). For negative $m$, we set $[U]_{m}^{n}=[U]_{0}^{n}$.

See for example Wallin [24, Lemma 2] for a proof of the following useful lemma.

Lemma 1.2. Let $Q(z) \neq 0$ be a polynomial of degree less than or equal to $M$. Then, for $|z| \leqslant R$,

$$
\sup _{|t| \leqslant R}\{|Q(t) / Q(z)|\} \leqslant\left(3 R_{0}\right)^{M} /\left|Q^{*}(z)\right|,
$$

where $R_{0}=\max \{1, R\}$ and $Q^{*}(z)=\prod_{i=1}^{n}\left(z-z_{i}\right)$ and where $z_{1}, \ldots, z_{n}$ are the zeroes of $Q(z)$ in $\{z|| z \mid \leqslant 2 R\}$.

2. Error formulae. The following is the analogue of the well-known contour integral error formula for classical Padé approximants.

THEOREM 2.1. Let $C$ be the boundary of a connected open set $\mathbf{D}$, and assume the finite interval I is contained in D. Let $f(z)$ be analytic in $I$, and let $f(z)$ and $\hat{f}(z)$ be meromorphic in $\mathbf{D}$. Let $L, M$ be nonnegative integers such that $M \geqslant \mu$. Let $S \in \mathbf{P}_{p}$, for some $p \leqslant M-\mu$, be such that both $S(z) f(z)$ and $S(z) \hat{f}(z)$ are analytic in $\mathbf{D}$ and on $C$.

(a) For all $z \in \mathbf{D}$,

$$
\begin{aligned}
f(z)-[L / M](z) & \\
& =\frac{1}{2 \pi i} \int_{C}\left\{\frac{\left(f(t) Q_{M}(t) \zeta_{1}(t, z)+\hat{f}(t) \hat{Q}_{M}(t) \zeta_{2}(t, z)\right) S(t)}{\left(Q_{M}(z) S(z)(t-z)\right)}\right\} d t .
\end{aligned}
$$

Here

$$
\zeta_{k}(t, z)=\left(R_{1}(z) / R_{1}(t)+(-1)^{k-1} R_{2}(z) / R_{2}(t)\right) / 2,
$$

for $k=1,2$, and $R_{1}(z)$ and $R_{2}(z)$ belong to $\mathbf{P}_{L+M+1-\mu}$, with all their zeroes in $I$.

(b) If $f(z)$ is real in $I$, then for all $z \in \mathbf{D}$,

$$
\begin{aligned}
f(z)-\langle L / M\rangle(z) & \\
& =\frac{1}{2 \pi i} \int_{C} f(t) Q_{M}^{*}(t) S(t) R(z) /\left(Q_{M}^{*}(z) S(z) R(t)(t-z)\right) d t,
\end{aligned}
$$

where $R \in \mathbf{P}_{L+M+1-\mu}$ and has all its zeroes in $I$.

(c) If $f(z)$ is not necessarily real in $I$, but if $f(z)$ and $\hat{f}(z)$ are analytic in $\mathbf{D}$, then for all $z \in \mathbf{D}$,

$$
\begin{aligned}
& f(z)-\langle L / M\rangle(z) \\
& \quad=\frac{1}{2 \pi i} \int_{C}\left(f(t) Q_{M}^{*}(t) \zeta_{3}(t, z)+\hat{f}(t) \hat{Q}_{M}^{*}(t) \zeta_{4}(t, z)\right) /\left(Q_{M}^{*}(z)(t-z)\right) d t .
\end{aligned}
$$

Here,

$$
\zeta_{k}(t, z)=\left(R_{3}(z) / R_{3}(t)+(-1)^{k-1} R_{4}(z) / R_{4}(t)\right) / 2,
$$

for $k=3,4$, and $R_{3}(z)$ and $R_{4}(z)$ belong to $\mathbf{P}_{L+1-\mu}$ with all their zeroes in $I$.

Proof. (a) Set $\Delta(z)=Q_{M}(z) f(z)-P_{L}(z)$. As the $\phi_{j}(z)$ are real on $I,(1.4)$ in Definition 1.1 implies that both $\Delta(z)$ and $\hat{\Delta}(z)$ are orthogonal to $\phi_{j}(z), 0 \leqslant j \leqslant L+$ $M$. Then for $k=1,2, \delta_{k}(z)=\left(\Delta(z)+(-1)^{k-1} \hat{\Delta}(z)\right) / i^{k-1}$ is real and analytic in $I$, 
and orthogonal to $\phi_{j}(z)$ for $0 \leqslant j \leqslant L+M$. By Lemma 1.1, there exist polynomials $R_{k}, k=1,2$, in $\mathbf{P}_{L+M+1-\mu}$ with all their zeroes in $I$ such that $\delta_{k}(z) / R_{k}(z)$ is analytic in $I, k=1,2$. Further we see $S(z) \delta_{k}(z)$ is analytic in $\mathbf{D}, k=1,2$. Then Cauchy's integral formula gives, for all $z \in \mathbf{D}$ and $k=1,2$,

$$
\begin{aligned}
& S(z) \delta_{k}(z) / R_{k}(z)=\frac{1}{2 \pi i} \int_{C} S(t) \delta_{k}(t) /\left(R_{k}(t)(t-z)\right) d t \\
& \quad=\frac{1}{2 \pi i} \int_{C} S(t)\left\{f(t) Q_{M}(t)+(-1)^{k-1} \hat{f}(t) \hat{Q}_{M}(t)\right\} /\left(i^{k-1} R_{k}(t)(t-z)\right) d t
\end{aligned}
$$

Here we have used the fact that $S(t) P_{L}(t) /\left(R_{k}(t)(t-z)\right)$ is analytic as a function of $t$ outside $\mathbf{D}$, and is $O\left(|t|^{-2}\right)$ as $|t| \rightarrow \infty$, since its numerator has degree $\leqslant p+L \leqslant L$ $+M-\mu$, while the denominator has degree exactly $L+M+2-\mu$. Solving for $\delta_{k}(z)$ from (2.6) and using $\Delta(z)=\left[\delta_{1}(z)+i \delta_{2}(z)\right] / 2$, gives (2.1) and (2.2).

(b) Here $f,\langle L / M\rangle$ are real in $I$, so by (1.4) and Lemma $1.1, f-\langle L / M\rangle$ has at least $L+M+1-\mu$ zeroes in $I$. The result follows as before.

(c) Here we again define $\Delta(z)=Q_{M}^{*}(z) f(z)-P_{L}^{*}(z)$ and see that $\Delta(z)$ and $\hat{\Delta}(z)$ are orthogonal to $\phi_{j}(z), 0 \leqslant j \leqslant L$. The proof is completed as in (a).

The following is the analogue of formulae developed by Nuttall [17] and Wallin [23] for classical Padé approximants.

THEOREM 2.2. Let $f(x)$ be such that all the moments $f_{j}=\int_{I} f(x) \phi_{j}(x) d \alpha(x)$, $j=0,1,2, \ldots$, exist. Let $H_{m}^{n}(w, z)=\sum_{j=m}^{n} \phi_{j}(w) \phi_{j}(z)$ for $0 \leqslant m \leqslant n<\infty$. Let $S \in$ $\mathbf{P}_{p}$ and let the nonnegative integers $L, M$ satisfy $M \geqslant 2 p$.

(a) $\operatorname{For}[L / M](z)=P_{L}(z) / Q_{M}(z)$,

$$
\begin{aligned}
e[L / M ; z] & =[S f]^{L+2 M-p}(z) / S(z)-[L / M](z) \\
& =\left[[S f]_{L-p+1}^{L-p+2 M} Q_{M}\right]_{L+M+1-p}^{L+3 M-p}(z) /\left(S(z) Q_{M}(z)\right) \\
& =\int_{I}[S f]_{L-p+1}^{L-p+2 M}(x) Q_{M}(x) H_{L+M+1-p}^{L+3 M-p}(x, z) d \alpha(x) /\left(S(z) Q_{M}(z)\right) .
\end{aligned}
$$

(b) $\operatorname{For}\langle L / M\rangle(z)=P_{L}^{*}(z) / Q_{M}^{*}(z)$,

$$
\begin{aligned}
e\langle L / M ; z\rangle & =[f]^{L+M}(z)-\langle L / M\rangle(z) \\
& =\left[[f]_{L-M+1}^{L+M} Q_{M}^{*}\right]_{L+1}^{L+2 M}(z) / Q_{M}^{*}(z) .
\end{aligned}
$$

Proof. (a) We have

$$
\begin{aligned}
V & =[S f]^{L+2 M-p} Q_{M}-S P_{L} \\
& =S\left(f Q_{M}-P_{L}\right)+\left([S f]^{L+2 M-p}-S f\right) Q_{M} .
\end{aligned}
$$

Now by Definition $1.1, f Q_{M}-P_{L}$ is orthogonal to $\phi_{k}, 0 \leqslant k \leqslant L+M$, so $S\left(f Q_{M}-P_{L}\right)$ is orthogonal to $\phi_{k}, 0 \leqslant k \leqslant L+M-p$. Similarly $[S f]^{L+2 M-p}-S f$ is orthogonal to $\phi_{k}, 0 \leqslant k \leqslant L+2 M-p$, so $\left([S f]^{L+2 M-p}-S f\right) Q_{M}$ is orthogonal to $\phi_{k}, 0 \leqslant k \leqslant L+M-p$. Hence $V$ is orthogonal to $\phi_{k}, 0 \leqslant k \leqslant L+M-p$, so

$$
V=[V]_{L+M-p+1}=\left[[S f]^{L+2 M-p} Q_{M}\right]_{L+M-p+1}
$$


(as $S P_{L}$ has degree at most $L+p \leqslant L+M-p$, and by (2.9))

$$
=\left[[S f]_{L-p+1}^{L+2 M-p} Q_{M}\right]_{L+M-p+1}^{L+3 M-p},
$$

(as $[S f]^{L-p} Q_{m}$ has degree at most $L+M-p$ and $[S f]^{L+2 M-p} Q_{M}$ has degree at most $L+3 M-p)$. The first part of (2.7) follows easily upon dividing by $S Q_{M}(z)$ in (2.10). The second part of (2.7) follows by substitution for $H_{L+M+1-p}^{L+3 M-p}(x, z)$.

(b) is very similar to (a).

3. Convergence of diagonal sequences. The following is the analogue of the Nuttall-Pommerenke Theorem for classical Padé approximants $[17,18]$.

THEOREM 3.1. Let $f(z)$ and $\hat{f}(z)$ be analytic in $\mathbf{C} \backslash E$ where $\operatorname{cap}(E)=0$. We assume $I \subset \mathbf{C} \backslash E$. If I is unbounded, then we require first $E$ to be bounded, secondly $I$ to be only semi-infinite, thirdly $f(z)$ to have at most a pole of finite order at infinity, and fourthly all moments of $f$ to exist. Let $\left\{\left[L_{k} / m_{k}\right]\right\}$ be a yiven sequence of linear Padé approximants to $f$ such that, for some $\lambda \geqslant 1$,

$$
1 / \lambda \leqslant M_{k} / L_{k} \leqslant \lambda \text { all } k \geqslant 1,
$$

and $\lim _{k \rightarrow \infty} L_{k}=\infty$. Then

(a) Given $r, \varepsilon, \delta$ all positive, there exists $k_{0}$ such that, for all $k \geqslant k_{0}$,

$$
\left|f(z)-\left[L_{k} / M_{k}\right](z)\right|<\varepsilon^{L_{k}+M_{k}},
$$

for $|z| \leqslant r$ except on a set $E_{k}$ such that $\operatorname{cap}\left(E_{k}\right)<\delta$. If $G$ is a compact subset of $\mathbf{C} \backslash E$ containing no limit points of poles of $\left\{\left[L_{k} / M_{k}\right]\right\}$, then (3.2) holds uniformly in $G$ for large $k$, that is $G \cap E_{k}=\phi$ for large $k$.

(b) There exists a subsequence $\left\{\left[L_{k}^{\prime} / M_{k}^{\prime}\right]\right\}$ of $\left\{\left[L_{k} / M_{k}\right]\right\}$ such that

$$
\lim _{k \rightarrow \infty}\left|f-\left[L_{k}^{\prime} / M_{k}^{\prime}\right]\right|(z)^{1 /\left(L_{k}^{\prime}+M_{k}^{\prime}\right)}=0,
$$

all $z \in \mathbf{C} \backslash F$ where $\operatorname{cap}(F)=0$.

(c) Any pole of $f$ of order $n$ is the limit of at least $n$ poles of $\left[L_{k} / M_{k}\right]$ counting multiplicities, as $k \rightarrow \infty$. Any nonisolated singularity or isolated essential singularity is the limit of poles of $\left[L_{k} / M_{k}\right]$ whose total multiplicity $\rightarrow \infty$ as $k \rightarrow \infty$.

Proof. (a) This follows using equations (2.1) and (2.2). Following, we provide the details.

Case 1. E, I bounded. Choose $r>1$ such that $E, I \subset\{z|| z \mid \leqslant r\}$. Choose $0<\eta$ $<1$ such that $\operatorname{dist}(E, I) \geqslant 3 \eta$, where $\operatorname{dist}(E, I)$ is the distance between $E, I$. Let $0<\gamma<\eta$ be given. Since $\operatorname{cap}(E)=0$, we can choose an integer $l \geqslant 1$ and $T \in \mathbf{P}_{l}$ such that

$$
E \subset\left\{z|| T(z) \mid<\gamma^{\prime}\right\}=\varrho_{1}(l, \gamma) .
$$

Using $\gamma<\eta$, it is easy to see that we can assume all $T$ 's zeroes lie at most $\eta$ from $E$, and hence lie at a distance of at least $2 \eta$ from $I$. Define $C=C_{1} \cup C_{2}$ where

$$
C_{1}=\left\{t|| T(t) \mid=\gamma^{l}\right\} ; \quad C_{2}=\{t|| t \mid=R\},
$$

with $R>r+\eta$. Since $t \in C_{1}$ implies $t$ is at most a distance $\gamma$ from one of $T$ 's zeroes, we see

$$
\operatorname{dist}(C, I) \geqslant \eta \text {. }
$$


From here on, we shall drop the subscripts $k$ from $L_{k}$ and $M_{k}$. From (2.1), (2.2) and from (3.5), (3.6), we have for $i=1,2$,

$$
\begin{aligned}
& \max _{t \in C_{1}}\left|\zeta_{i}(t, z)\right| \leqslant((c+r) / \eta)^{L+M+1-\mu} \quad \text { all }|z| \leqslant r \\
& \left.\max _{t \in C_{2}}\left|\zeta_{i}(t, z)\right| \leqslant((c+r) /(R-c))^{L+M+1-\mu} \quad \text { all }|z| \leqslant r\right\},
\end{aligned}
$$

where $I=(a, b)$ and $c=\max \{|a|,|b|\}$. Next choose $\omega>\lambda$. Choose $S=T^{n}$ where $n$ is the greatest integer $\leqslant(M-\mu) /(\omega l)$. Then for $M$ large enough, $S$ has degree $p=n l$, where

$$
M /(2 \omega) \leqslant p \leqslant(M-\mu) / \omega<M-\mu .
$$

Let $1>\theta>\gamma$ be given. Then $\varrho_{1}(l, \theta)$ contains $\mathscr{L}_{1}(l, \gamma)$ in its interior, hence there exists positive $\Delta$ such that $z \notin \mathcal{L}_{1}(l, \theta)$ implies $|t-z| \geqslant \Delta$ for all $t \in C_{1}$. Next, Lemma 1.2 shows that there exists a lemniscate $\varrho_{2}(M, \theta)$ with foci at the zeroes of $Q_{M}$ in $\{z|| z \mid \leqslant 2 R\}$ such that

$$
\sup _{|t| \leqslant R}\left|Q_{M}(t) / Q_{M}(z)\right| \leqslant(3 R / \theta)^{M} \quad \text { all }|z| \leqslant R, z \notin \mathcal{L}_{2}(M, \theta) .
$$

Obviously, we can replace $Q_{M}(t)$ by $\hat{Q}_{M}(t)$ in the left-hand side of (3.9). Lemma 1.2 also shows

$$
\sup _{|t| \leqslant R}|S(t) / S(z)| \leqslant(3 R / \theta)^{P} \quad \text { all }|z| \leqslant R, z \notin \mathcal{L}_{1}(l, \theta)
$$

where again $S(t)$ may be replaced by $\hat{S}(t)$. Further

$$
\sup _{t \in C_{1}}|S(t) / S(z)| \leqslant(\gamma / \theta)^{p} \quad \text { all } z \notin \mathcal{L}_{1}(l, \theta) .
$$

We now use (3.5) to (3.11) to bound the right-hand side of (2.1). For all $|z| \leqslant r$, such that

$$
z \notin E_{M}=\left\{\varrho_{1}(l, \theta) \cup \varrho_{2}(M, \theta)\right\} \cap\{z|| z \mid \leqslant r\}
$$

we obtain

$$
\begin{aligned}
|f-[L / M]|(z) \leqslant K\{ & ((c+r) / \eta)^{L+M+1-\mu}(3 R / \theta)^{M}(\gamma / \theta)^{P} \\
& \left.+((c+r) /(R-c))^{L+M+1-\mu}(3 R / \theta)^{M}(3 R / \theta)^{P}\right\},
\end{aligned}
$$

(where $K$ is a constant depending only on $f, \hat{f}, C_{1}, C_{2}, \gamma$ and $\theta$ )

$$
\leqslant K^{\prime}\left\{\tau_{1}^{M}+\tau_{2}^{M}\right\}
$$

where

$$
\tau_{1}=((c+r) / \eta)^{1+\lambda}(3 R / \theta)(\gamma / \theta)^{1 /(2 \omega)}
$$

and

$$
\tau_{2}=((c+r) /(R-c))^{1+1 / \lambda}(3 R / \theta)^{1+1 / \omega}
$$

and $K^{\prime}$ depends only on $f, \hat{f}, C_{1}, C_{2}, \mu, r, \theta, \eta, R$. We have used (3.1) and (3.8) here. Next, using $\operatorname{cap}(\mathcal{L}(\cdot, \theta)) \leqslant \theta$ and the well-known inequality $\operatorname{cap}\left(F_{1} \cup F_{2}\right) \leqslant$ $\left(d\left(F_{1} \cup F_{2}\right) \max _{i} \operatorname{cap}\left(F_{i}\right)\right)^{1 / 2}$, we see $\operatorname{cap}\left(E_{M}\right) \leqslant((2 r) \theta)^{1 / 2}<\delta$ if $\theta$ is chosen small 
enough, $\theta$ being independent of $M$. Having chosen $\theta$, and noting that $c, r, \lambda, \omega, \eta$ were chosen in advance, we can choose $R$ so large that the term $\tau_{2}$ is less than $(\varepsilon / 2)^{1+\lambda}$ (possible as the power of $R$ in $\tau_{2}$ is $-1 / \lambda+1 / \omega<0$ ). Having chosen $R$, we can choose $\gamma$ so small that $\gamma<\min \{\theta, \eta\}$ and so that the term $\tau_{1}$ is less than $(\varepsilon / 2)^{1+\lambda}$. The estimate (3.2) then follows from (3.12).

Case 2. Either $E$ or $I$ is unbounded. Using a device due to Wallin [24, Theorem 4, p. 444] this problem may be reduced to a case very similar to Case 1 . Let $r, \varepsilon, \delta$, all positive, be given, and choose $d \in \mathbf{R} \backslash(I \cup E)$ such that $|d| \gg r$. Consider the bilinear transformation $w=1 /(z-d) \Leftrightarrow z=1 / w+d$. I and $E$ in the $z$-plane are mapped to $\tilde{I}$ and $\tilde{E}$ in the $w$-plane such that:

(1) $\tilde{E}$ is compact and $\tilde{I}$ is a finite real interval.

(2) $\tilde{I} \cap \tilde{E}=\varnothing$ since only one of $I$ and $E$ is unbounded.

(3) $\operatorname{cap}(\tilde{E})=0$ (see Wallin [24, Lemma 3b, p. 442]).

Further, if $I$ is unbounded, then $w=0 \in \tilde{I}$ and $f(z)$ has at most a pole of finite-order-say order $\nu$-at $z=\infty$. If $I$ is bounded, define $\nu=0$. Then in either case $\tilde{f}(w)=w^{\nu} f(1 / w+d)$ is analytic in $\mathbf{C} \backslash \tilde{E}$. Let $N=\max \{L, M\}$ and $\tilde{P}_{L}(w)=$ $w^{N+\nu} P_{L}(1 / w+d)$ and $\tilde{Q}_{M}(w)=w^{N} Q_{M}(1 / w+d)$. Then $\tilde{\Delta}(w)=\tilde{f}(w) \tilde{Q}_{M}(w)-$ $\tilde{P}_{L}(w)=w^{N+\nu} \Delta(1 / w+d)$ where $\Delta(z)=f(z) Q_{M}(z)-P_{L}(z)$. Since $\operatorname{Re}(\Delta(z))$ and $\operatorname{Im}(\Delta(z))$ have at least $L+M=1-\mu$ zeroes in $I$, it follows that $\operatorname{Re}(\tilde{\Delta}(w))$ and $\operatorname{Im}(\tilde{\Delta}(w))$ have at least $L+M+1-\mu$ zeroes in $\tilde{I}$, since $w$ is real when $z$ is real. With this observation, we can derive formulas for $\tilde{f}(w)-\tilde{P}_{L}(w) / \tilde{Q}_{M}(w)$ as in Theorem 2.1, with the following differences: Note that the degree of $\tilde{P}_{L}(w)$ is at most $N+\nu$ and degree of $\tilde{Q}_{M}(w)$ is at most $N$ and $L+M \geqslant(1+1 / \lambda) N$, which follows from (3.1). Then in (2.1), $R_{i}, i=1,2$, are chosen to be in $\mathbf{P}_{s}$, where $s=$ greatest integer $\leqslant(1+1 / \lambda) N+1-\mu$. Further we choose $S \in \mathbf{P}_{p}$ where $p \leqslant$ $N / \lambda-\mu$. As for the convergence proof, we proceed as in Case 1, but choose $S(w)=T^{n}(w)$ where $n=$ greatest integer $\leqslant(N / \lambda-\mu) /(\omega l)$. See Theorem 1 in [15] for the modifications in a similar situation. For (b) and (c), see the proof of Theorem 1 in [15].

Remarks. (a) When $f$ is nonreal in $\mathbf{R}$, the linear (and nonlinear) Padé approximants do not interpolate to $f$, so Theorem 3.1(a) is not contained in Theorem 4 of Wallin [24].

(b) It is no restriction to assume both $f$ and $\hat{f}$ are analytic outside $E$. If $f$ has singularities $E_{1}$ of capacity zero, then $f$ and $\hat{f}$ are analytic outside $E=E_{1} \cup \bar{E}_{1}$ and $\operatorname{cap}(E)=0$.

(c) As in Karlsson [12], Theorem 3.1 holds for a slightly larger class of functions.

THEOREM 3.2. Let $f(z), \hat{f}(z), I$ and $E$ be as in Theorem 3.1 and assume (3.1) holds. If $f$ is nonreal in $I$, we require $E=\varnothing$ and $\liminf _{k \rightarrow \infty} L_{k} / M_{k}>1$. Then the results stated in parts (a), (b), (c) of Theorem 3.1 hold with $[L / M]$ replaced by $\langle L / M\rangle$.

Proof. This follows from Theorem 2.1(b),(c) in much that same way Theorem 3.1 follows from Theorem 2.1(a).

4. Convergence of nondiagonal sequences. Suetin [22] stated a de Montessus de Balloré type theorem for linear Padé approximants, as well as convergence in capacity of general nondiagonal sequences of linear Padé approximants, provided 
$\alpha^{\prime}(x)$ is positive almost everywhere in the finite interval $I .^{2}$ The contour integrals (2.1), (2.3), (2.4) enable one to prove convergence in capacity of nondiagonal sequences without such a restriction on $\alpha$, but only in a much smaller region than the ellipse considered by Suetin (cf. Theorem 3, 4 in [15]). For simplicity we restrict ourselves in this section to functions meromorphic in $\mathbf{C}$.

THEOREM 4.1. Let I be a bounded real interval. Let $f$ be meromorphic in $\mathbf{C}$ with poles of total multiplicity $q(0 \leqslant q \leqslant \infty)$. Let $\left\{L_{k}\right\}$ and $\left\{M_{k}\right\}$ satisfy $\lim _{k \rightarrow \infty}\left(M_{k}+1\right) / L_{k}$ $=0$. Further let $\lim _{k \rightarrow \infty} M_{k}=\infty$ if $q=\infty$; but let $\liminf _{k \rightarrow \infty} M_{k} \geqslant p^{\prime}+\mu$ if $q<\infty$, where $p^{\prime}(\leqslant 2 q)$ is the smallest integer such that for some $S_{1} \in \mathbf{P}_{p}$ both $S_{1}(z) f(z)$ and $S_{1}(z) \hat{f}(z)$ are entire. For the nonlinear Padé approximants, we place the additional restrictions that either $f$ be real in $I$ or $q=0$.

Let $U_{k}(z)$ denote either $\left[L_{k} / M_{k}\right](z)$ or $\left\langle L_{k} / M_{k}\right\rangle(z)$. Then:

(a) Given $r, \varepsilon, \delta$ all positive, there exists $k_{0}$ such that, for $k \geqslant k_{0}$,

$$
\left|f(z)-U_{k}(z)\right|<\varepsilon^{L_{k}} \quad \text { all }|z|<r, z \notin F_{k}=\mathcal{L}\left(2 M_{k}, \delta^{L_{k} / M_{k}}\right),
$$

so that $\lim _{k \rightarrow \infty} \operatorname{cap}\left(F_{k}\right)=0$.

If $G$ is a compact subset of $\mathbf{C}$ containing no poles of $f$, or limit points of poles of $\left\{U_{k}\right\}$, then the estimate in (4.1) holds uniformly in $G$ for large $k$, that is, $G \cap F_{k}=\varnothing$ for large $k$.

$$
\lim _{k \rightarrow \infty}\left|f(z)-U_{k}(z)\right|^{1 / L_{k}}=0 \quad \text { all } z \in \mathbf{C} \backslash E
$$

where

$$
E \subset \limsup _{k} \mathcal{L}\left(2 M_{k}, \delta_{k}^{L_{k} / M_{k}}\right) \text { and } \lim _{k \rightarrow \infty} \delta_{k}=0 .
$$

(c) If $z_{0}$ is a pole of $f$ of order $n$, then $z_{0}$ is the limit of at least $n$ poles counting multiplicity of $\left\{U_{k}(z)\right\}$ as $k \rightarrow \infty$.

(d) If $\mu=0$ and $p^{\prime}=q<\infty$ and $M_{k}=q$ for large $k$, then $\left\{U_{k}\right\}$ converges uniformly and geometrically to $f$ in compact subsets of $\mathbf{C}$ containing no poles of $f$.

Proof. (a) Consider the linear Padé approximants first. Let $C=\{t|| t \mid=R\}$ where $R>r$. Choose the smallest integer $p$ such that for some $S \in \mathbf{P}_{p}$ both $S(z) f(z)$ and $S(z) \hat{f}(z)$ are analytic in $|z| \leqslant R$. Let us drop the subscripts $k$ in $L_{k}$ and $M_{k}$. Then (2.1) and (2.2) hold. For $i=1,2$, we see

$$
\max _{t \in C}\left|\zeta_{i}(t, z)\right| \leqslant((r+c) /(R-c))^{L+M+1-\mu} \text { all }|z| \leqslant r
$$

where $I=(a, b)$ and $c=\max \{|a|,|b|\}$. Then we obtain, using (2.1) and Lemma 1.2 ,

$$
|f-[L / M]|(z) \leqslant K((r+c) /(R-c))^{L+M}\left(3 R / \delta^{L / M}\right)^{M+p}
$$

all $|z| \leqslant r, z \notin \mathcal{L}\left(M+p, \delta^{L / M}\right)$ where $K$ is a constant independent of $L, M, z$ and the lemniscate $\mathcal{L}$ has foci at the zeroes of $S Q_{M}$ in $\{z|| z \mid \leqslant 2 R\}$. The result follows if we choose $R$ large relative to $r+c$ and to $1 / \delta$, and as $M+p \leqslant M+p^{\prime} \leqslant 2 M$ for

\footnotetext{
${ }^{2}$ However the referee has informed the authors that his proofs are incomplete.
} 
large $M$. The proof for the nonlinear Padé approximants follows similarly from Theorems 2.1 (b), (c).

(b) follows from (a).

(c) follows from (a)- see [15, Theorems 3,4$]$.

(d) follows from (a), (b), (c) and the restrictions on $M, p^{\prime}$.

REMARKs. (a) Although sets of the form (4.3) may seem unaesthetic, there is no simpler way of writing the exceptional set in the general case. See [14] for a study of such sets. It is well known that when $\lim _{k \rightarrow \infty} M_{k} \log k / L_{k}=0, E$ satisfying (4.3) has Hausdorff dimension zero, and when $\Sigma_{k}\left(M_{k}+1\right) / L_{k}<\infty, E$ satisfying (4.3) has logarithmic capacity zero.

(b) One consequence of Theorem 4.1(d) is that if $d \alpha(x)$ is of one sign in $I$, even if it vanishes identically in some subinterval of $I$, then the orthogonal expansions to entire functions $f$ converge uniformly to $f$ in compact subsets of $\mathbf{C}$. A similar result holds for functions analytic in certain finite domains containing $I$ (cf. Theorems 3,4 in [15]). A second consequence of Theorem 4.1 (d) is that if $f$ has poles of finite total multiplicity $p$ in $\mathbf{C}$ and if the poles are symmetric with respect to the real axis (true for example if $f$ is real in $\mathbf{R}$ ) then $[L / p] \rightarrow f(z)$ as $L \rightarrow \infty$ except at $f$ 's poles, provided only $d \alpha(x)$ is of one sign in $I$.

5. Wallin-type theorems. In this section, we prove results analogous to those of Wallin [23], using Theorem 2.2. In addition to (1.1), we assume that

$$
\left|\phi_{j}(z)\right| \leqslant(\Gamma(z))^{n},
$$

in $\mathbf{C}$, where $\Gamma(z) \geqslant 1$, is uniformly bounded in compact subsets of $\mathbf{C}$, and is independent of $n$. In particular, when $I$ is bounded and $\alpha^{\prime}(x)$ is positive almost everywhere in $I$, it follows from Theorem 3.7.4 in Freud [7, p. 123] and the maximum modulus principle, that (5.1) holds.

Theorem 5.1. Let $f(x)$ be as in Theorem 2.2 with the notation therein. Let $S \in \mathbf{P}_{p}$ and define $b_{j}=\int_{I} f(x) S(x) \phi_{j}(x) d \alpha(x), j=0,1,2 \ldots$ Let $\left\{\left[L_{k} / M_{k}\right]\right\}$ be such that $\liminf \operatorname{in}_{k \rightarrow \infty} M_{k} \geqslant 2 p$ and define

$$
N_{k}=\left\{\begin{array}{l}
\max \left\{M_{k}, L_{k}\right\} \quad \text { all } k \geqslant 1 \text { if } \underset{k \rightarrow \infty}{\limsup L_{k} / M_{k}=\infty,} \\
M_{k} \quad \text { all } k \geqslant 1 \text { if } \underset{k \rightarrow \infty}{\limsup } L_{k} / M_{k}<\infty .
\end{array}\right.
$$

Set

$$
\rho_{k}=\max \left\{\left|b_{j}\right| \mid L_{k}-p<j \leqslant L_{k}+2 M_{k}-p\right\}^{1 / N_{k}} \quad \text { all } k \geqslant 1
$$

and assume

$$
\lim _{k \rightarrow \infty} \rho_{k}=0 .
$$

(a) Given $r>1,0<\eta<1, \varepsilon>0$, there exists a positive integer $k_{0}$ such that, for $k \geqslant k_{0}$,

$$
\left|e\left[L_{k} / M_{k} ; z\right]\right|<\rho_{k}^{(1-\eta) N_{k}}
$$


all $|z| \leqslant r, z \notin F_{k}=\varrho\left(M_{k}+p, \varepsilon\right)$ so $\operatorname{cap}\left(F_{k}\right) \leqslant \varepsilon$. Here $e[L / M ; z]$ is defined by (2.7).

(b) If a monotone increasing sequence $\left\{c_{k}\right\}$ satisfies

$$
\lim _{k \rightarrow \infty} c_{k}=\infty \text { but } \lim _{k \rightarrow \infty} \rho_{k} c_{k}=0,
$$

then

$$
\lim _{k \rightarrow \infty}\left|e\left[L_{k} / M_{k} ; z\right]\right|^{1 /\left(L_{k}+M_{k}\right)}=0 \quad \text { all } z \in \mathbf{C} \backslash E,
$$

where

$$
E \subset \limsup _{k \rightarrow \infty} \mathcal{L}\left(M_{k}, c_{k} \rho_{k}\right) .
$$

In particular, when $\lim _{k \rightarrow \infty}[S f]^{k}(z)=(S f)(z)$ in $\mathbf{D} \subset \mathbf{C}$ (where $f(z)$ continues $f(x)$ analytically from $I$ to $\mathbf{D})$ then $\lim _{k \rightarrow \infty}\left[L_{k} / M_{k}\right](z)=f(z)$ all $z \in \mathbf{D} \backslash E$.

Proof. (a) We shall drop the subscripts $k$ from $L_{k}, M_{k}$, and so on. Using the second part of (2.7), the definition of $H_{m}^{n}(x, z)$, using Definition 1.5 and (5.1), (5.3), we see

$$
|e[L / M ; z]| \leqslant 4\left(\int_{I} d|\alpha|(x)\right) M^{2}\left(\Gamma_{1} \Gamma(z)\right)^{5(L+M)} \rho^{N} \sup _{t \in I}\left|Q_{M}(t) / Q_{M}(z)\right| /|S(z)|
$$

where $\Gamma_{1}=\sup \{\Gamma(t) \mid t \in I\}$. Now Lemma 1.2 shows that

$$
\sup _{t \in I}\left|Q_{M}(t) / Q_{M}(z)\right| /|S(z)| \leqslant(3 r)^{M} / \varepsilon^{M+P},
$$

all $|z| \leqslant r, z \notin F=\mathfrak{L}(M+p, \varepsilon)$, this lemniscate having foci at the zeroes of $S Q_{M}(z)$ in $|z| \leqslant 2 r$. Finally, we note that (5.2) implies that there exists $\lambda>0$ such that $N \geqslant \lambda L$, while $N \geqslant M$. The result now follows from (5.4), (5.9), (5.10) and the uniform boundedness of $\Gamma(z)$ in compact subsets of $\mathbf{C}$.

(b) Let $\left\{r_{k}\right\}$ be an increasing sequence of positive numbers such that $\lim _{k \rightarrow \infty} r_{k}=$ $\infty$ and let $\Gamma_{k}=\sup \left\{\Gamma(z)|| z \mid \leqslant r_{k}\right\}$ all $k>1$. Then (5.9) and Lemma 1.2 give for some constant $d$ independent of $k$, and for all $|z| \leqslant r_{k}, z \notin \mathcal{L}\left(M, c_{k} \rho_{k}\right)$,

$$
\begin{aligned}
|e[L / M ; z]| & \leqslant\left(d \Gamma_{k}\right)^{5(L+M)} \rho_{k}^{N}\left(3 r_{k} /\left(c_{k} \rho_{k}\right)\right)^{M} /|S(z)| \\
& \leqslant\left\{\left(d \Gamma_{k}\right)^{5(1+1 / \lambda)} 3 r_{k} / c_{k}\right\}^{N} /|S(z)|,
\end{aligned}
$$

(since $N \geqslant M$ and $N \geqslant \lambda L$ and $r_{k} /\left(c_{k} \rho_{k}\right)>1$ for large $k$, by (5.6)). If we choose $r_{k}$ to tend to $\infty$ sufficiently slowly with $k$, we obtain (5.7) and (5.8) as $\lim _{k \rightarrow \infty} c_{k}=\infty$. The finitely many zeroes of $S(z)$ can be included in the lim sup in (5.8) by changing some sparse subsequence of foci of the lemniscates in (5.8).

THEOREM 5.2. Let $f$ be as in Theorem 2.2 with the notation therein. Let $\left\{\left\langle L_{k} / M_{k}\right\rangle\right\}$ be given and let $\left\{N_{k}\right\}$ be defined by (5.2). Further let

$$
\rho_{k}=\max \left\{\left|f_{j}\right| \mid \max \left\{L_{k}-M_{k}+1,0\right\} \leqslant j \leqslant L_{k}+M_{k}\right\}^{1 / N_{k}} \text { all } k \geqslant 1,
$$

and assume (5.4) holds. Then (a), (b) in Theorem 5.1 hold, if we replace $e[L / M ; z]$ by $e\langle L / M ; z\rangle$ and set $p=0, S=1$. 
Proof. The proof is similar to Theorem 5.1, but uses Theorem 2.2(b).

Corollary 5.3. Assume $I=[-1,1]$ and that $\alpha^{\prime}(x)$ is positive almost everywhere in I. Let $f(z)$ be analytic on $I$, and let $\mathcal{E}$ be the largest ellipse with foci at $-1,1$ and major and minor axes respectively on the real and imaginary axes, such that $f$ has poles of finite total multiplicity $p$ inside $\mathcal{E}$. Let $\left\{L_{k}\right\},\left\{M_{k}\right\},\left\{\rho_{k}\right\},\left\{c_{k}\right\}$ be as in Theorem 5.1. Let $2 r$ be the sum of $\mathcal{E}$ 's major and minor axes. Let $D(z)=z+\left(z^{2}-1\right)^{1 / 2}$, the branch of $\left(z^{2}-1\right)^{1 / 2}$ being chosen to be real and positive for real $z>1$. Then

$$
\limsup _{k \rightarrow \infty}\left|f-\left[L_{k} / M_{k}\right]\right|(z)^{1 /\left(L_{k}+M_{k}\right)} \leqslant|D(z)| / r,
$$

all $z$ inside $\mathcal{E}, z \notin E$ where $E$ satisfies (5.8). A similar result holds for nonlinear Padé approximants and the case $p=0$.

Proof. Choose $S \in \mathbf{P}_{p}$ such that $(f S)(z)$ is analytic inside $\mathcal{E}$. Then

$$
\limsup _{k \rightarrow \infty}\left|(f S)(z)-[f S]^{k}(z)\right|^{1 / k} \leqslant|D(z)| / r
$$

inside $\varepsilon$.

See Freud [7, Theorem 3.7.4].

Corollary 5.4. Let $f,\left\{L_{k}\right\},\left\{M_{k}\right\},\left\{N_{k}\right\},\left\{\rho_{k}\right\}$ be as in Theorem 5.1 or Theorem 5.2 .

(a) If for some $\alpha>0, \Sigma_{k} \rho_{k}^{\alpha}<\infty$, then $E$ in (5.7) and (5.8) has $\alpha$-dimensional Hausdorff measure zero, that is $t^{\alpha}-m(E)=0$.

(b) If for some $\gamma>1, \Sigma_{k}\left(\log 1 / \rho_{k}\right)^{-\gamma}<\infty$, then $E$ in (5.7) and (5.8) has Hausdorff $(\log 1 / t)^{-\gamma}-m(E)=0$.

(c) If $\sum_{k}\left(\log 1 / \rho_{k}\right)^{-1}<\infty$, then $E$ in (5.7) and (5.8) has $\operatorname{cap}(E)=0$.

Proof. (a) One can choose monotone increasing $\left\{c_{k}\right\}$ satisfying (5.6) and $\Sigma_{k}\left(c_{k} \rho_{k}\right)^{\alpha}<\infty$. Then the $\alpha$-dimensional form of Cartan's lemma [1,p. 194] shows that $E$ has $\alpha$-dimensional Hausdorff measure zero.

(b), (c) are similar to (a).

When $d \alpha(x)$ is of one sign in $I$, the theorems of this section hold for unbounded intervals without further restrictions. This may be seen by modifying (5.9).

\section{REFERENCES}

1. G. A. Baker, Jr., Essential of Padé approximants, Academic Press, New York, 1975.

2. E. W. Cheney, Introduction to approximation theory, McGraw-Hill, New York, 1966.

3. J. S. R. Chisholm and A. K. Common, Padé-Chebyshev approximants, Padé Approximation and its Applications (L. Wuytack, ed.), Lecture Notes in Math., vol. 765, Springer-Verlag, Berlin, 1979, pp. 1-19.

4. C. W. Clenshaw and K. Lord, Rational approximations from Chebyshev series, Studies in Numerical Analysis (B. K. P. Scaife, ed.), Academic Press, New York, 1974, pp. 95-113.

5. J. Fleischer, Analytic continuation of scattering amplitudes and Padé approximants, Nuclear Phys. B 37 (1972), 59-76.

6. __ Nonlinear Padé approximants for Legendre series, J. Math. Phys. 14 (1973), 246-248.

7. G. Freud, Orthogonal polynomials, Pergamon Press, Budapest, 1971.

8. A. A. Gončar, On the convergence of generalized Padé approximants of meromorphic functions, Math. USSR-Sb. 27 (1975), 503-514.

9. W. B. Gragg, Laurent, Fourier and Chebyshev Padé tables, Padé and Rational Approximation (E. B. Saff, R. S. Varga, eds.), Academic Press, New York, 1977, pp. 61-72. 
10. E. Hille, Analytic function theory, vol. 2, Ginn, Boston, Mass., 1962.

11. J. T. Holdeman, A method for the approximation of functions defined by formal series expansions in orthogonal polynomials, Math. Comp. 23 (1969), 275-287.

12. J. Karlsson, Rational interpolation and best rational approximation, J. Math. Anal. Appl. 53 (1976), $38-52$.

13. D. S. Lubinsky, Diagonal Padé approximants and capacity, J. Math. Anal. Appl. 78 (1980), 58-67.

14. __ Exceptional sets of Padé approximants, Ph.D. Thesis, Witwatersrand University, Johannesburg, 1980 .

15. appear).

16. H. J. Maehly, Rational approximations for transcendental functions, Proceedings of the International Conference on Information Processing, Butterworths, 1960.

17. J. Nuttall, The convergence of Padé approximants of meromorphic functions, J. Math. Anal. Appl. 31 (1970), 147-153.

18. C. Pommerenke, Padé approximants and convergence in capacity, J. Math. Anal. Appl. 41 (1973), $775-780$.

19. A. Sidi, Computation of the Chebyshev-Pade table, J. Comput. Appl. Math. 1 (1975), 69-71.

20. Uniqueness of Padé approximants from series of orthogonal polynomials, Math. Comp. 39 (1977), 738-739.

21. H. Stahl, Beiträge zum Problem der Konvergenz von Padéapproximierenden, $\mathrm{Ph}$. D. Thesis, Technische Universität, Berlin, 1976.

22. S. P. Suetin, On the convergence of rational approximations to polynomial expansions in domains of meromorphy, Math. USSR.-Sb. 34 (1978), 367-381.

23. H. Wallin, The convergence of Padé approximants and the size of the power series coefficients, Appl. Anal. 4 (1974), 235-251.

24. H. Wallin, Potential theory and approximation of analytic functions by rational interpolation, Proceedings of Complex Analysis Conference at Joensuu, (I. Laine, O. Lehto, T. Sorvali, eds.), Lecture Notes in Math., vol. 747, Springer-Verlag, Berlin, 1979, pp. 434-450.

Department of Computer Science, Technion-IsRael Institute of TeChNology, Haifa, IsRael

Faculty of Mathematics, Technion-IsRael Institute of Technology, Haifa, IsRael 